\title{
Exome sequencing of fetal anomaly syndromes: novel phenotype-genotype discoveries
}

\author{
Nicole Meier ${ }^{1,2,3}$ - Elisabeth Bruder ${ }^{3,4}$ - Olav Lapaire ${ }^{5}$ Irene Hoesli ${ }^{5}$ Anjeung Kang ${ }^{6}$ Jürgen Hench ${ }^{4}$. \\ Sylvia Hoeller ${ }^{3,4} \cdot$ Julie De Geyter $^{1} \cdot$ Peter Miny $^{1,3} \cdot$ Karl Heinimann $^{1,3} \cdot$ Rabih Chaoui $^{7} \cdot$ Sevgi Tercanli ${ }^{3,6}$. \\ Isabel Filges ${ }^{1,2,3}$
}

Received: 14 July 2018 / Revised: 2 October 2018 / Accepted: 4 December 2018 / Published online: 24 January 2019

(c) The Author(s) 2019. This article is published with open access

\begin{abstract}
The monogenic etiology of most severe fetal anomaly syndromes is poorly understood. Our objective was to use exome sequencing (ES) to increase our knowledge on causal variants and novel candidate genes associated with specific fetal phenotypes. We employed ES in a cohort of 19 families with one or more fetuses presenting with a distinctive anomaly pattern and/or phenotype recurrence at increased risk for lethal outcomes. Candidate variants were identified in 12 families (63\%); in 6 of them a definite diagnosis was achieved including known or novel variants in recognized disease genes (MKS1, $O T X 2, F G F R 2$, and $R Y R 1)$ and variants in novel disease genes describing new fetal phenotypes (CENPF, KIF14). We identified variants likely causal after clinical and functional review (SMAD3, KIF4A, and PIGW) and propose novel candidate genes (PTK7, DNHD1, and TTC28) for early human developmental disease supported by functional and crossspecies phenotyping evidence. We describe rare and novel fetal anomaly syndromes and highlight the diagnostic utility of ES, but also its contribution to discovery. The diagnostic yield of the future application of prenatal ES will depend on our ability to increase our knowledge on the specific phenotype-genotype correlations during fetal development.
\end{abstract}

Supplementary information The online version of this article (https:// doi.org/10.1038/s41431-018-0324-y) contains supplementary material, which is available to authorized users.

\section{Isabel Filges}

Isabel.filges@usb.ch

Isabel.filges@unibas.ch

1 Medical Genetics, Institute of Medical Genetics and Pathology, University Hospital Basel, Basel, Switzerland

2 Department of Clinical Research, University Hospital Basel, Basel, Switzerland

3 University of Basel, Basel, Switzerland

4 Pathology, Institute of Medical Genetics and Pathology, University Hospital Basel, Basel, Switzerland

5 Department of Obstetrics and Gynecology, University Hospital Basel, Basel, Switzerland

6 Centre for Prenatal Ultrasound, Freie Strasse, Basel, Switzerland

7 Centre for Prenatal Diagnosis, Friedrichstrasse, Berlin, Germany

\section{Introduction}

Birth defects are the leading cause of perinatal lethality in industrialized countries [1]. With the advance of highresolution ultrasound fetal structural anomalies are now detected increasingly early during pregnancy, raising questions about diagnosis, etiology, prognosis, and recurrence risk for parents and health care providers, particularly in the presence of more than one fetal anomaly likely indicating a congenital malformation syndrome. Prenatal high-resolution chromosomal microarray analysis will allow a diagnosis of causal copy number variants in up to additional $10 \%$ of pregnancies after the exclusion of the frequent aneuploidies, but 80 $90 \%$ of families remain without definite diagnosis. Targeted molecular testing may be indicated if a specific monogenic entity is suspected based on clinical signs. The monogenic etiology of phenotypes observed postnatally has been successfully investigated by using genome-wide sequencing technologies [2]. Exome or genome approaches have now become available in routine clinical genetics services for the diagnosis of patients with developmental disorders. Only recently, however, the 
delineation of fetal anomaly phenotypes received increasing attention including the discussion of introducing exome sequencing (ES) in standard prenatal care. Best et al. [3] discussed its promises and pitfalls including the review of 31 studies with series of five or more fetuses and diagnostic rates varying between $6.2 \%$ and $80 \%$. Approaches in such studies are highly heterogeneous ranging from prospective prenatal ES to the study of selected cases with severe fetal anomalies after termination or stillbirth [4]. In general, the diagnostic yield seems to be higher in fetuses with multiple congenital anomalies and in selected series with detailed clinical genetics review [3]. However, a significant proportion of anomaly phenotypes encountered during the antenatal developmental stages may be specific to fetal life since they will lead to embryonic, fetal or perinatal lethality and will have escaped etiological research and clinical delineation so far. They may also represent an incomplete or severe allelic presentation of a phenotype described to occur postnatally, and the diagnosis therefore can remain unrecognized at this stage of development. Filges and Friedman [5] stressed the value of applying genomic sequencing to examine such rare extreme phenotypes despite the challenges to expect when interpreting the clinical significance of variants and proving their causality. Based on these considerations we explored the clinical and molecular diagnosis using ES in a series of 19 families with one or more fetuses with severe structural anomalies. We aimed at identifying causal as well as candidate variants, showing the utility of ES for diagnosis but also discovery, and sought to contribute to the further delineation and etiology of phenotype presentations of known and novel multiple congenital anomaly syndromes in fetal stages intending to increase the yield of future prenatal diagnostic ES.

\section{Patients and methods}

The study was approved by the Ethics Commission Northwest Switzerland (EKNZ 2014-174). After written informed consent for participation was obtained from the parents, we prospectively recruited 19 families - 26 fetuses (after fetal or perinatal death or from terminated pregnancies) and one child - that presented with severe anomalies of unexplained etiology initially identified by ultrasound scanning. Families were included in the study if (i) the fetus showed a pattern of two or more anomalies associated with a high risk for fetal or perinatal lethality that suggested a genetic disorder or (ii) there was familial recurrence of the fetal anomaly phenotype and if (iii) there were detailed clinical fetal ultrasound and/or autopsy data available and (iv) high-resolution chromosomal microarray did not show a causal chromosomal anomaly or copy number variant. Families were recruited through the Medical Genetics Clinic at the University Hospital Basel, Switzerland. The fetal phenotypes were reviewed by experienced clinical geneticists, fetal and neuropathologists, and maternal-fetalmedicine specialists. Autopsy was performed in affected fetuses of 18 families.

\section{Exome sequencing (ES) and variant prioritization}

Genomic DNA was used from previous prenatal specimen extracted from chorionic villus sampling, amniocentesis or extracted from fresh frozen or formalin fixed paraffin embedded (FFPE) fetal tissue. Parental DNA was extracted from whole-blood samples. ES was performed in familial trios (or quattros, when available after recurrence). Library preparation (Agilent SureSelect ${ }^{\mathrm{XT}}$ Library Prep Kit) and exome capture using the Agilent SureSelect ${ }^{\mathrm{XT}}$ Human All Exon V6 (Agilent, Santa Clara, CA) was followed by paired-end read sequencing $(2 \times 100 \mathrm{bp}$ read length) on a HiSeq 2500 or HiSeq 4000 platform (Illumina, San Diego, CA) with an average coverage of $\times 100$. Quality estimation of the sequence reads was performed by generating quality control statistics with FastQC (http://www.bioinformatics. bbsrc.ac.uk/projects/fastqc). Illumina CASAVA (1.8.2) was used to demultiplex the sequencing reads. Adapter trimming was performed with Skewer (Version 0.2.2). Sequencing reads were mapped and aligned to the reference genome sequence (hg19) with Burrows-Wheeler Aligner (BWAmem Version 0.7.2). Alignments with a Phred quality score below 30 for the entire read and potential PCR duplicates were discarded using samtools (Version 0.1.18). To increase sensitivity, variant calling was performed with samtools and varscan (Version 2.3.5). Variants with a coverage $\leq 10 \mathrm{X}$ and not supported by at least 4 reads $(20 \%)$ were discarded. Familial segregation of variants was performed to identify de novo, autosomal recessive and $\mathrm{X}$ linked inheritance. Variants were filtered on a heterozygous population frequency (GMAF) of $<5 \%$ in control databases (dbSNP142, 1000G, gnomAD), and absence of homozygosity in healthy individuals (ExAC) and classified as known or novel according to their presence or absence in curated databases. Variants were prioritized according to their potential to disrupt protein function using standard prediction tools (SIFT, Provean, Polyphen2, Mutationtaster, Human Splicing Finder v.3.0), amino acid conservation assessment across species (PhyloP, PhastCons) and the American College of Medical Genetics and Genomics (ACMG) variant classification guidelines [6]. In addition, we applied the HOPE protein prediction program (http://www.cmbi.ru.nl/hope/) which combines structural information including the 3D structure of the protein, if available, to analyze the specific mutational effect on the 


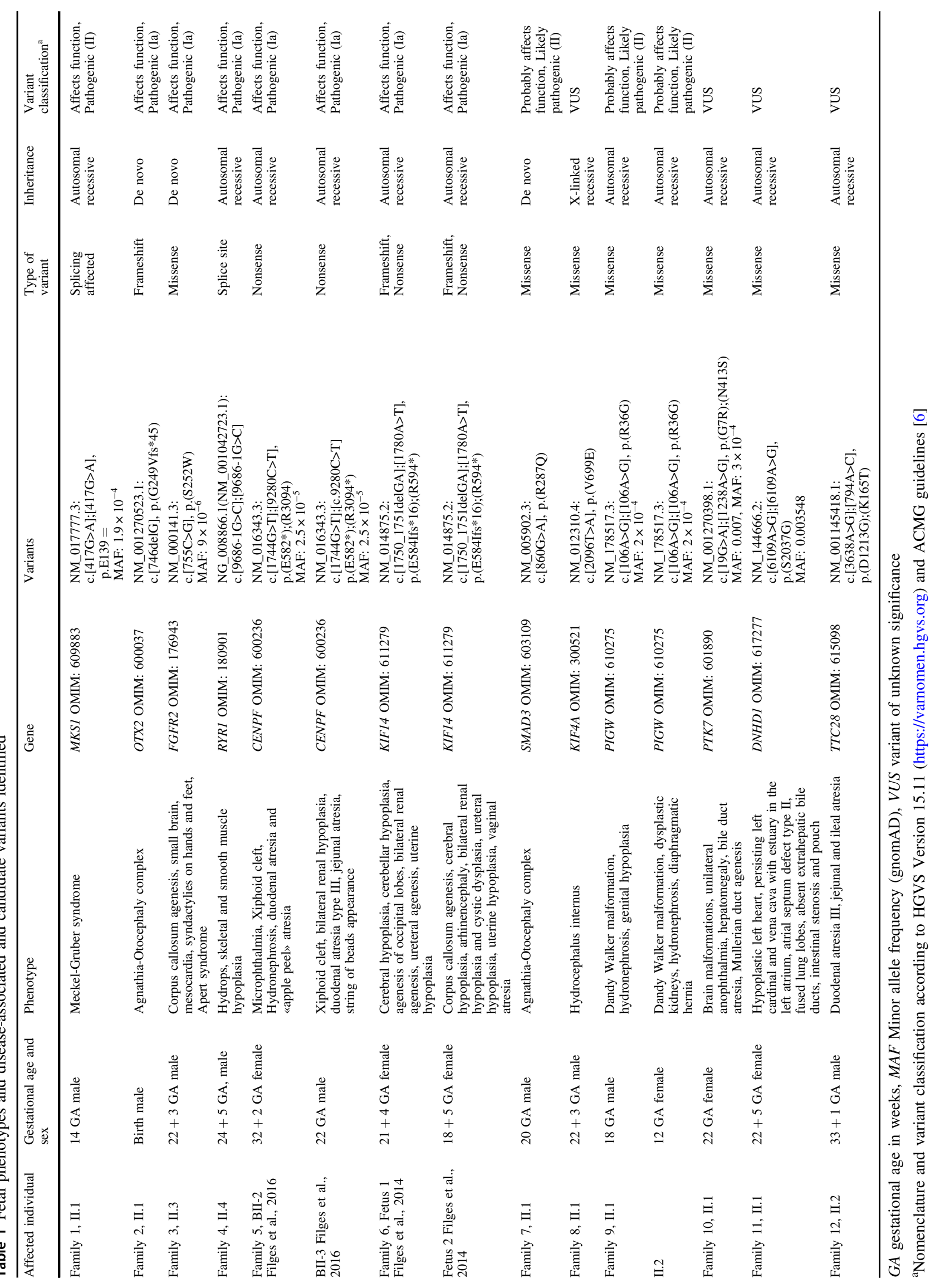


protein structure. Putative candidate variants were visually reanalyzed in each family using the Integrative Genomics Viewer (http://software.broadinstitute.org/software/igv/) and confirmed by Sanger sequencing (primer sequences are available on request). Genotype-phenotype correlations were considered critical for identification of the causal relationship between a candidate variant and anomaly phenotype. Existing information was compiled from medical literature and databases (Pubmed, OMIM, ClinVar, Decipher) and related protein networks and signaling pathways in embryonic development (Reactome, Uniprot, Ingenuity pathway analysis (Qiagen)). We systematically interrogated zebrafish, mouse, and drosophila phenotype databases (www.zfin.org; www.informatics.jax.org; www. mousephenotype.org; www.flybase.org) using cross-species phenotype comparison for validation of candidate genes. GeneMatcher (genematcher.org) was queried for similar cases. We used the HGVS nomenclature Version 15.11 to describe variant effects (https://varnomen.hgvs.org/). All variants identified were submitted to the LOVD database (https://databases.lovd.nl/shared/individuals 00181103-09, 00181141, 00181143-49).

\section{Functional studies}

In 3 fetuses, FFPE tissue from affected organs was used against age-matched control fetal FFPE tissue for reverse transcription analysis of the candidate gene to assess the presence or absence of the transcript. Control tissue was obtained from fetuses without structural anomalies confirmed by autopsy after intrauterine fetal death likely due to asphyxia. RNA from FFPE tissue specimens was isolated with the RecoverAll ${ }^{\mathrm{TM}}$ Total Nucleic Acid Isolation Kit for FFPE (Ambion, Thermo Fisher Scientific, Waltham, Massachusetts). Reverse transcription (RT) of mRNA was performed with the High-Capacity cDNA Reverse Transcription Kit (Applied Biosystems, Waltham, MA, USA). 18S RNA primers served as quality control. In 2 fetuses, additional qPCR was used to confirm the lower concentration of the transcript. qPCR was performed with the SYBR Green Master Mix (Applied Biosystems, Waltham, MA, USA). Expression levels of the transcripts were normalized to GUSB expression.

\section{Results}

Phenotypes and causal or candidate variants, if identified, are summarized in Table 1 . Supplementary Table S1 shows the unsolved cases. The frequency of congenital anomalies in different organ systems is displayed in Supplementary Table S2. We identified variants that would cause or potentially explain the fetal anomaly phenotype in 12 out of
19 families (63\%). A definite diagnosis was achieved in $50 \%$ of those ( 6 families), while in the remaining 6 families variants had to be classified as variants of unknown significance according to the ACMG guidelines [6]. Variants in 12 different genes were identified (RYR1, MKS1, FGFR2, PIGW, CENPF, KIF14, SMAD3, KIF4A, PTK7, DNHD1, $T T C 28$, and $O T X 2)$. Variants that were classified to affect or likely affect function were detected in 8 different genes (RYR1, MKS1, FGFR2, PIGW, CENPF, KIF14, SMAD3, and $O T X 2)$. The variants identified are categorized into the following groups: (1) a priori diagnostic, since they were known or novel in known disease genes explaining the fetal anomaly phenotype, (2) confirmed to be disease-associated by the identification of additional families and/or functional analysis, and thus led to the description of a new disease gene and related phenotype, (3) likely disease-associated by clinical and functional review in (a) a known disease gene or (b) a new candidate gene.

\section{Known or novel variants in disease genes explaining fetal anomalies}

We identified variants that affect or likely affect protein function in MKS1, OTX2, FGFR2, and RYR1. The homozygous variant in $M K S 1$ was previously reported to affect splicing [7] and explained a recurrent Meckel-Gruber syndrome-like phenotype in family 1 . Variants in OTX2 are reported to cause agnathia-otocephaly in rare instances [8]. Although novel, the de novo frameshift variant (c. [746delG], p.(G249Vfs*45)) was considered to affect function, causing the typical phenotype of agnathiaotocephaly in family 2 . In a fetus with bilateral syndactyly of hands and feet and corpus callosum agenesis (family 3 ), the molecular diagnosis of Apert syndrome was made by identifying a known pathogenic de novo variant in FGFR2 [9]. For family 4, we confirmed previously that a novel homozygous splice-site variant in the skeletal ryanodine receptor 1 gene (RYRI) causes lethal hydrops and severe skeletal and smooth muscle hypoplasia [10].

\section{Variants in novel genes confirmed to be causal for new fetal phenotypes}

In family 5, previously reported by Filges et al. [11], novel compound heterozygous truncating variants in CENPF were identified in a fetus with a suggested clinical diagnosis of Strømme syndrome and a sibling with a severe malformation phenotype reminiscent of a ciliopathy phenotype (c. [1744G>T];[c.9280C >T], p.(E582*);(R3094)). The identification of variants in the same gene in unrelated families and functional zebrafish results confirmed causality and a variable phenotype presentation ranging from viable Strømme syndrome to a lethal fetal ciliopathy [11, 12]. 
Novel compound heterozygous truncating variants were identified in KIF14 (c.[1750_1751delGA];[1780A>T], p. (E584Ifs*16);(R594*)) in two fetuses with a ciliary phenotype not compatible with any described syndrome in family 6 [13]. This was the first report of a human phenotype caused by bi-allelic truncating variants in KIF14. The authors suggested KIFI4 to be a candidate gene for allelic viable phenotypes including isolated microcephaly, which was now confirmed in several patients harboring diseaseassociated variants in KIF14 as well [14].

\section{Candidate variants-likely disease-associated by clinical and functional review}

In 6 families potential candidate variants were identified in genes for which other variants are known to cause a postnatal phenotype that, however, significantly differs from the phenotype observed in the fetus (SMAD3, KIF4A, PIGW) or that are currently not known to cause a human phenotype at all (TTC28, PTK7, DNHD1). They were prioritized due to their role in distinct pathways and/or similar phenotypes in animal models.

\section{Genes in which novel candidate variants may cause a fetal phenotype different from the postnatal phenotype}

In family 7 , fetal ultrasound identified agnathia-otocephaly complex with complete absence of the mandible and a submandibular position of the ears, cleft palate, and aortic isthmus stenosis. No variants were identified in $O T X 2$ and $P R R X 1$ through ES and additional Sanger sequencing, in which disease causing variants were previously reported to cause the agnathia-otocephaly phenotype $[8,15]$. The novel de novo missense variant in SMAD3 was prioritized (c. [860G $>\mathrm{A}]$, p.(R287Q)) out of four remaining candidates (KRT86, PRICKLE1, MYH3, SMAD3) since SMAD3, OTX2 and PRRX1 are reported to act in the same signaling pathway implicated in the development of the branchial arches [16]. Variants in SMAD3 were previously reported to cause autosomal dominant Loeys-Dietz syndrome 3 (OMIM: 613795), a connective tissue disorder, presenting with aortic aneurysm, cardiac anomalies, cleft palate and significant micro-/retrognathia. Proteins of the SMAD family play an important role in transcriptional regulation. The variant we identified is located in the MH2-domain of SMAD3 important for the interaction between the different SMAD proteins (Uniprot). According to the HOPE prediction, the mutated residue may disturb the binding properties of the MH2 domain since it cannot form hydrogen bonds and salt bridges. Further functional studies on chicken embryos to prove causality are ongoing.

Among 3 variants identified in different genes (OSGINl, $K I F 4 A, B C A T 1)$ potentially disease-causing according to prediction algorithms we considered the $\mathrm{X}$-linked novel hemizygous missense variant in the KIF4A gene (c. [2096T>A], p.(V699E)) likely to be associated with the isolated hydrocephalus in the male fetus of family 8 . The variant was also present in the healthy mother, consistent with X-linked recessive inheritance. Variants in KIF4A are reported to cause $\mathrm{X}$-linked intellectual disability (OMIM: 300923) [17]. However, KIF4A is a motor protein that translocates PRC1, a cytokinesis protein, to the ends of the spindle microtubules during mitosis [18], regulates the PARP1 activity in brain development and the survival of neurons [19] and is a member of the L1CAM recycling pathway. Variants in L1CAM are well known to cause $\mathrm{X}$ linked isolated and syndromic hydrocephalus [20]. The novel variant is in the highly conserved PRC1 interacting domain and is predicted to disrupt interaction. qPCR confirmed a significant reduction of KIF4A mRNA in brain tissue of the affected fetus (12\%) compared to FFPE brain tissue of an age-matched control (Supplementary Figure S1). The low mRNA levels indicate nonsense-mediated decay (NMD) and a likely loss-of-function mechanism.

In family 9 both fetuses presented with a Dandy-Walker malformation, hydronephrosis, dysplastic kidneys, and genital hypoplasia, and an additional diaphragmatic hernia in one fetus, suggesting a clinical diagnosis of Fryns- or Fryns like syndrome. In total, variants in 6 genes were considered (ABCA1, AIMIL, CTDSP2, NOP16, RSU1, and $P I G W$ ). A homozygous missense variant in PIGW (c.[106 $A>G] ;[106 \quad A>G], p .(R 36 G)$ that segregated in both fetuses was prioritized because phenotypes caused by variants in $P I G V$ and PIGN genes of the PIG family show a phenotypic overlap with Fryns syndrome [21] or are described to be causal for the Fryns phenotype [22]. The presence of PIGW is required in the early steps of GPI anchor biosynthesis, and bi-allelic variants in genes encoding components of the GPI anchor biogenesis pathway have been suggested to be a rare cause of variable developmental and malformation phenotypes [23]. So far, variants in $P I G W$ were reported to cause a glycosylphosphatidylinositol biosynthesis defect 11 (OMIM 610275) presenting with developmental delay, intellectual disability, and seizures [24]. The variants in patients described are closer to the $3^{\prime}$-end of the gene than the one in the fetuses with an anomaly phenotype we identified. This variant is located in the transmembrane domain and the mutant amino acid is predicted to disturb the transport activity of the protein (HOPE).

\section{Novel candidate genes}

The structural anomalies of the fetus of family 10, including multiple brain anomalies, spina bifida, unilateral anophthalmia, bile duct atresia, agenesis of the Müllerian ducts, hepatomegaly and bilateral cleft palate was not specific for 
any previously described congenital anomaly syndrome. From the two genes with potentially disease-associated biallelic variants (MTHFDIL, PTK7) we selected compound heterozygous missense variants in PTK7 (c.[19G>A]; [c.1238 A>G], p.(G7R);(N413S)) as the best candidates since two PTK7 loss of function mouse models show severe neural tube defects, cystic kidneys, micrognathia, and unilateral anophthalmia $[25,26]$. No human phenotype has been described so far, but PTK7 deficiency is suspected to play a role in neural tube defects [27], being a key protein in the embryonic Wnt and PCP signaling pathways. Furthermore, PTK7 regulates the outgrowth of the Wolffian duct [28], which is essential for the formation of the Müllerian duct [29]. One of the detected variants (c.19G>A) is located in a transcript (NM_001270398.1), that is coding for the protein isoform e which has an alternative exon 1 that lacks a signal peptide sequence for localization in the cell membrane. Currently, functional properties of this isoform are investigated. RT-PCR on RNA extracted from liver FFPE tissue of the affected fetus and tissue of an agematched control showed a significant signal decrease in the affected fetus. qPCR of the same samples confirmed a decrease of mRNA expression to 6\% (Supplementary Figure S2). These findings are indicative of NMD of the PTK7 mRNA due to aberrant splicing, at least in the liver and for this isoform.

In family 11, the fetus had a complex heart defect with a hypoplastic left heart and aorta ascendens, subtotal mitral valve atresia, persistent cardinal vein and atrial septal defect, fused lung lobes, gallbladder agenesis, and reduced intrahepatic bile ducts, incomplete intestinal rotation and a pouch-like extension of the proximal jejunum. The malformation pattern was suggestive of a heterotaxy/ciliopathy phenotype. ES detected potentially disease-associated variants in three different genes (CACNA1A, PLCH2, $D N H D 1)$. A homozygous missense variant in DNHD1 (c.[6109 A>G];[6109 A>G], p.(S2037G)) was prioritized due to its function in the dynein heavy chain, although no consanguinity was reported. Variants in other genes such as DNAL1 (OMIM: 610062), DNAII (OMIM: 604366) and DNAH11 (OMIM: 603339), which are coding for dynein compartments, are known to cause situs inversus-like phenotypes. Little is known about the exact function of DNHD1. It was reported as a candidate gene for intellectual disability [30]. Family 12 presented with recurrence of intestinal atresia. DNA was only available from one child for trio analysis. ES detected compound heterozygosity in 4 different genes (CASZ1, PLEKHM2, COL5A1, and TTC28), but only variants in TTC28 (c.[3638 A>G];[c.794 A>C], p.(D1213G);(K265T)) were predicted disease associated and were highly conserved across species. We prioritized TTC28 because variants in TTC7A (OMIM: 609332), a member of the same gene family, causes autosomal recessive gastrointestinal defects, and variants in $T T C 21 B$ (OMIM: 612014) are reported in human ciliopathies.

\section{Discussion}

The overall primary detection rate of disease-associated variants in known disease genes explaining the fetal phenotype is $21 \%$ in our highly selected series of fetuses with structural anomalies. For those families counseling to inform about prognosis and recurrence risk as well as reproductive choices, such as prenatal diagnosis or preimplantation genetic diagnosis, in further pregnancies is available. Other studies report detection rates of $10-21 \%$ $[4,31-33]$, however, inclusion criteria and approaches in all studies including ours are highly heterogeneous and therefore restrict comparability. Our detection rate increases after having ascertained disease-association for the variants in the novel candidate genes KIFI4 and CENPF by the investigation of additional unrelated affected patients as well as functional and animal model studies [11, 13]. Whereas clinical diagnoses such as agnathia-otocephaly and MeckelGruber syndrome were obvious, the presentation of Apert syndrome was atypical since craniosynostosis, the key clinical sign in the corresponding postnatal phenotype, cannot be appreciated at that early developmental stage and therefore hampered targeted molecular genetic testing. This reverse phenotyping highlights the importance of appreciating fetal phenotypes as a variable of developmental timing. Candidate variants identified in the additional 6 families do not have an immediate clinical impact, but may guide the future search for further affected patients and functional studies for confirmatory evidence. Particularly, the suspected causal relationships between variants in $P I G W$ for a Fryns syndrome phenotype, KIF4A as a player in the L1CAM pathway for X-linked hydrocephalus and variants in SMAD3 which may cause agnathia-otocephaly as the severe end of the Loeys-Dietz spectrum emphasize the importance of clinical and developmental genetics review in addition to formal variant assessment. Similarly, the phenotype of about one-third of the fetuses examined, including family 10,11 , and 12 , is reminiscent of some type of ciliopathy compatible with the fundamental role of pathways implied in ciliogenesis and ciliary function in embryonic development. Nine of the twelve conditions identified are likely following autosomal recessive inheritance, which may in part reflect recruiting bias including families with phenotype recurrence. However, embryonically lethal mouse models suggest that autosomal recessive inheritance may play an important role in phenotypes with early lethality. The identification of KIF14 variants causing a lethal ciliary anomaly pattern highlights the significance of investigating such extreme phenotypes 
for the primary description of a human disease gene, representing the severe phenotypic spectrum of an allelic viable postnatal disorder. Large-scale projects such as the International Mouse Phenotyping Consortium (IMPC) producing knockout mouse lines will be an important resource to support the characterization of novel human disease genes. In four of the seven families in which we were not able to delineate a compelling candidate gene, fetuses presented with recurrence of an isolated anomaly (Supplementary Table S1). This supports the observation of others as well as previous prenatal microarray studies for $\mathrm{CNVs}$ that a monogenic etiology is more likely identified in the presence of multiple anomalies. Besides the multiple limitations in fetal phenotyping and exome analysis, multifactorial or polygenic inheritance or epigenetic mechanisms remain a possible explanation. Much more research is needed to explore the utility of genome-wide sequencing in isolated anomalies, particularly when there is phenotype recurrence, potentially also suggestive of a recessive disorder with incomplete phenotype presentation in the antenatal period. The recruitment of additional unrelated fetuses presenting with the same phenotype is one of the most important recognized challenges in elucidating pathogenicity of candidate variants in the prenatal field despite an arising awareness of the importance to investigate such families. Prenatal phenotypes may be imprecise, and autopsy often not done for various reasons impairing phenotype-genotype comparisons and the collection of a larger number of similarly affected fetuses. Functional analysis of potential candidate variants and the generation of appropriate variant specific animal models demands expertize and significant resources. The current diagnostic yield of prenatal ES, however, may remain limited when the interpretation of variants continues to rely on experiences with postnatal patients exclusively. Recruiting patients in a perinatal setting may raise high parental expectations, and a thorough discussion of the current limitations in the interpretation of private variants is required. However, using ES as a discovery tool integrating the delineation of precise fetal phenotypes we contribute to further understand altered developmental pathways specific to fetal life. Recruitment of additional families and studies of variant effects will be mandatory to confirm causal relationships and understand disease mechanisms. Besides the gain of knowledge on biological mechanisms in early human development, this will allow increasing the utility of prenatal ES in a longterm perspective, and much more families will benefit from diagnostic certainty in this sensitive field.

Supplementary information regarding this article is available at (https://www.nature.com/ejhg/)

Acknowledgements We would like to thank the families for their gracious participation and our colleagues Deborah Bartholdi, MD,
Gwendolin Manegold-Brauer, MD and Viviane Cina, MSc for referring single cases.

Funding The Swiss National Science Foundation (SNSF), Project Grant to Isabel Filges (320030_160200) supported this work.

\section{Compliance with ethical standards}

Conflict of interest The authors declare that they have no conflict of interest.

Publisher's note: Springer Nature remains neutral with regard to jurisdictional claims in published maps and institutional affiliations.

Open Access This article is licensed under a Creative Commons Attribution 4.0 International License, which permits use, sharing, adaptation, distribution and reproduction in any medium or format, as long as you give appropriate credit to the original author(s) and the source, provide a link to the Creative Commons license, and indicate if changes were made. The images or other third party material in this article are included in the article's Creative Commons license, unless indicated otherwise in a credit line to the material. If material is not included in the article's Creative Commons license and your intended use is not permitted by statutory regulation or exceeds the permitted use, you will need to obtain permission directly from the copyright holder. To view a copy of this license, visit http://creativecommons. org/licenses/by/4.0/.

\section{References}

1. Osterman MJK, Kochanek KD, MacDorman MF, Strobino DM, Guyer B. Annual summary of vital statistics: 2012-2013. Pediatrics. 2015;135:1115-25.

2. Yang Y, Muzny DM, Reid JG, et al. Clinical whole-exome sequencing for the diagnosis of mendelian disorders. $\mathrm{N}$ Engl $\mathrm{J}$ Med. 2013;369:1502-11.

3. Best S, Wou K, Vora N, Van der Veyver IB, Wapner R, Chitty LS. Promises, pitfalls and practicalities of prenatal whole exome sequencing. Prenat Diagn. 2018;38:10-19.

4. Boissel S, Fallet-Bianco C, Chitayat D. et al. Genomic study of severe fetal anomalies and discovery of GREB1L mutations in renal agenesis. Genet Med. 2017. https://doi.org/10.1038/gim.2017.173.

5. Filges I, Friedman JM. Exome sequencing for gene discovery in lethal fetal disorders - harnessing the value of extreme phenotypes. Prenat Diagn. 2015;35:1005-9.

6. Richards S, Aziz N, Bale S, et al. Standards and guidelines for the interpretation of sequence variants: a joint consensus recommendation of the American College of Medical Genetics and Genomics and the Association for Molecular Pathology. Genet Med. 2015;17:405-23.

7. Consugar MB, Kubly VJ, Lager DJ, et al. Molecular diagnostics of Meckel-Gruber syndrome highlights phenotypic differences between MKS1 and MKS3. Hum Genet. 2007;121:591-9.

8. Patat O, van Ravenswaaij-Arts CMA, Tantau J, et al. Otocephalydysgnathia complex: description of four cases and confirmation of the role of OTX2. Mol Syndromol. 2013;4:302-5.

9. Wilkie AOM, Slaney SF, Oldridge M, et al. Apert syndrome results from localized mutations of $F G F R 2$ and is allelic with Crouzon syndrome. Nat Genet. 1995;9:165-72.

10. Meier N, Bruder E, Filges I. A novel homozygous splice-site mutation in RYR1 causes fetal hydrops and affects skeletal and smooth muscle development. Prenat Diagn. 2017;37:720-4. 
11. Filges I, Bruder E, Brandal K, et al. Strømme syndrome is a ciliary disorder caused by mutations in CENPF. Hum Mutat. 2016;37: 359-63.

12. Waters AM, Asfahani R, Carroll $\mathrm{P}$, et al. The kinetochore protein, $C E N P F$, is mutated in human ciliopathy and microcephaly phenotypes. J Med Genet. 2015;52:147-56.

13. Filges I, Nosova E, Bruder E, et al. Exome sequencing identifies mutations in KIF14 as a novel cause of an autosomal recessive lethal fetal ciliopathy phenotype. Clin Genet. 2014;86:220-8.

14. Makrythanasis P, Maroofian R, Stray-Pedersen A, et al. Biallelic variants in KIF14 cause intellectual disability with microcephaly. Eur J Hum Genet. 2018;26:330-9.

15. Dasouki M, Andrews B, Parimi P, Kamnasaran D. Recurrent agnathia-otocephaly caused by DNA replication slippage in. PRRX1 Am J Med Genet Part A. 2013;161:803-8.

16. Gekas J, Li B, Kamnasaran D. Current perspectives on the etiology of agnathia-otocephaly. Eur J Med Genet. 2010;53:358-66.

17. Willemsen $\mathrm{MH}, \mathrm{Ba} \mathrm{W}$, Wissink-Lindhout WM, et al. Involvement of the kinesin family members KIF4A and KIF5C in intellectual disability and synaptic function. J Med Genet. 2014;51:487-94.

18. Zhu C, Jiang W. Cell cycle-dependent translocation of PRC1 on the spindle by Kif4 is essential for midzone formation and cytokinesis. Proc Natl Acad Sci USA. 2005;102:343-8.

19. Midorikawa R, Takei Y, Hirokawa N. KIF4 motor regulates activity-dependent neuronal survival by suppressing PARP-1 enzymatic activity. Cell. 2006;125:371-83.

20. Basel-Vanagaite L, Straussberg R, Friez MJ, et al. Expanding the phenotypic spectrum of $L 1 C A M$-associated disease. Clin Genet. 2006;69:414-9.

21. Reynolds KK, Juusola J, Rice GM, Giampietro PF. Prenatal presentation of Mabry syndrome with congenital diaphragmatic hernia and phenotypic overlap with Fryns syndrome. Am J Med Genet Part A. 2017;173:2776-81.

22. McInerney-Leo AM, Harris JE, Gattas M, et al. Fryns syndrome associated with recessive mutations in PIGN in two separate families. Hum Mutat. 2016;37:695-702.

23. Pagnamenta AT, Murakami Y, Taylor JM, et al. Analysis of exome data for 4293 trios suggests GPI-anchor biogenesis defects are a rare cause of developmental disorders. Eur J Hum Genet. 2017;25:669-79.

24. Chiyonobu T, Inoue N, Morimoto M, Kinoshita T, Murakami Y. Glycosylphosphatidylinositol (GPI) anchor deficiency caused by mutations in $\mathrm{PIGW}$ is associated with West syndrome and hyperphosphatasia with mental retardation syndrome. J Med Genet. 2014;51:203-7.

25. Paudyal A, Damrau C, Patterson VL, et al. The novel mouse mutant, chuzhoi, has disruption of Ptk7 protein and exhibits defects in neural tube, heart and lung development and abnormal planar cell polarity in the ear. BMC Dev Biol. 2010;10:87.

26. San Agustin JT, Klena N, Granath K, et al. Genetic link between renal birth defects and congenital heart disease. Nat Commun. 2016;7:11103.

27. Wang M, De Marco P, Merello E, Drapeau P, Capra V, Kibar Z. Role of the planar cell polarity gene Protein tyrosine kinase 7 in neural tube defects in humans. Birth Defects Res Part A - Clin Mol Teratol. 2015;103:1021-7.

28. Xu B, Washington AM, Domeniconi RF, et al. Protein tyrosine kinase 7 is essential for tubular morphogenesis of the Wolffian duct. Dev Biol. 2016;412:219-33.

29. Chiga M, Ohmori T, Ohba T, Katabuchi H, Nishinakamura R. Preformed Wolffian duct regulates Müllerian duct elongation independently of canonical Wnt signaling or Lhx1 expression. Int J Dev Biol. 2014;58:663-8.

30. Anazi S, Maddirevula S, Faqeih E, et al. Clinical genomics expands the morbid genome of intellectual disability and offers a high diagnostic yield. Mol Psychiatry. 2017;22:615-24.

31. Carss KJ, Hillman SC, Parthiban V, et al. Exome sequencing improves genetic diagnosis of structural fetal abnormalities revealed by ultrasound. Hum Mol Genet. 2014;23:3269-77.

32. Yates CL, Monaghan KG, Copenheaver D, et al. Whole-exome sequencing on deceased fetuses with ultrasound anomalies: expanding our knowledge of genetic disease during fetal development. Genet Med. 2017;19:1171-8.

33. Drury S, Williams H, Trump N, et al. Exome sequencing for prenatal diagnosis of fetuses with sonographic abnormalities. Prenat Diagn. 2015;35:1010-7. 